\title{
Microbial Identification Certainty
}

National Cancer Institute

\section{Source}

National Cancer Institute. Microbial Identification Certainty. NCI Thesaurus. Code C102667.

A qualification that describes the degree of certainty of the correctness of a microbial identification test result. 